\title{
Ubiquitin Proteasome System Activity is Suppressed by Curcumin Following Exercise-Induced Muscle Damage in Human Skeletal Muscle
}

THOMAS D. CARDACI, STEVEN B. MACHEK, DYLAN T. WILBURN, PAUL S. HWANG, \& DARRYN S. WILLOUGHBY

Exercise \& Biochemical Nutrition Laboratory, Department of Health, Human Performance, \& Recreation, Robbins College of Health and Human Sciences, Baylor University, Waco, TX, USA

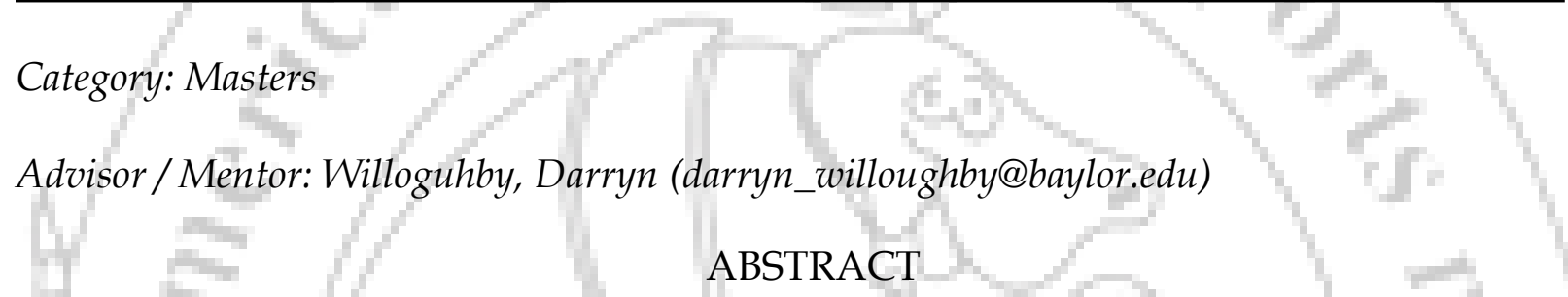

PURPOSE: Curcumin is a natural polyphenolic compound with antioxidant and anti-inflammatory properties. In vitro and in vivo animal studies have demonstrated that exposure to curcumin leads to dysregulation of the ubiquitin-proteasome system (UPS). However, to date, no study has investigated curcumin's ability to influence UPS activity in a human model. Therefore, the purpose of this study was to investigate the effects of curcumin supplementation on markers of UPS activity in the presence of elevations in UPS activity due to exercise-induced muscle damage. METHODS: Twenty-three recreationally active male and female participants between the ages of 18-30 were randomized into a curcumin (CUR; $\mathrm{n}=11$ ) or placebo (PLA; $=12$ ) group. Both groups were instructed to consume $2 \mathrm{~g}$ of their respective supplement and $20 \mathrm{mg}$ of piperine for 11 consecutive days. Following 8 consecutive days of supplementation, participants performed a 45 minute eccentrically-biased muscle damaging treadmill protocol at $60 \%$ VO2max. Muscle biopsies and delayed onset muscle soreness (DOMS) analyses were performed 30 minutes prior and 3, 24, 48, and 72 hours following exercise-induced muscle damage. Skeletal muscle ubiquitin, MAFbx/Atrogin-1, ubiquitin specific peptidase 19 (USP19), and chymotrypsinlike protease concentrations were measured using ELISA. A 3-way repeated measures ANOVA with pairwise comparisons was conducted with significance set at $p \leq 0.05$. RESULTS: Both groups had a significant time effect for DOMS $(\mathrm{p}<.001)$. Pairwise comparisons indicated DOMS was significantly greater from baseline at all time points except 72 hours following muscle damage. No significant differences were found for USP19 between groups. Regardless of time, a significant main effect for condition was observed for ubiquitin $(\mathrm{p}=.016)$ and MAFbx/Atrogin-1 $(\mathrm{p}=.006)$ where CUR was significantly lower than PLA. Additionally, a significant main effect for gender was observed for $\mathrm{MAFbx} /$ Atrogin-1 ( $\mathrm{p}=.013)$ where females were greater than males. A significant group $\mathrm{x}$ gender interaction was found for chymotrypsin-like protease $(p=.049)$ where males had lower values with curcumin supplementation while females had slightly higher. CONCLUSION: Curcumin supplementation in humans does appear to dysregulate UPS activity in the presence of exercise-induced muscle damage. Specifically, curcumin's ability to suppress protein ubiquination provides preliminary evidence of curcumin supplementation's potential therapeutic role in decreasing protein degradation associated with skeletal muscle damage and perhaps other atrophic scenarios. 